\title{
THE MILKY WAY GALAXY
}

Edited by HUGO VAN WOERDEN, RONALD J. ALLEN and W. BUTLER BURTON
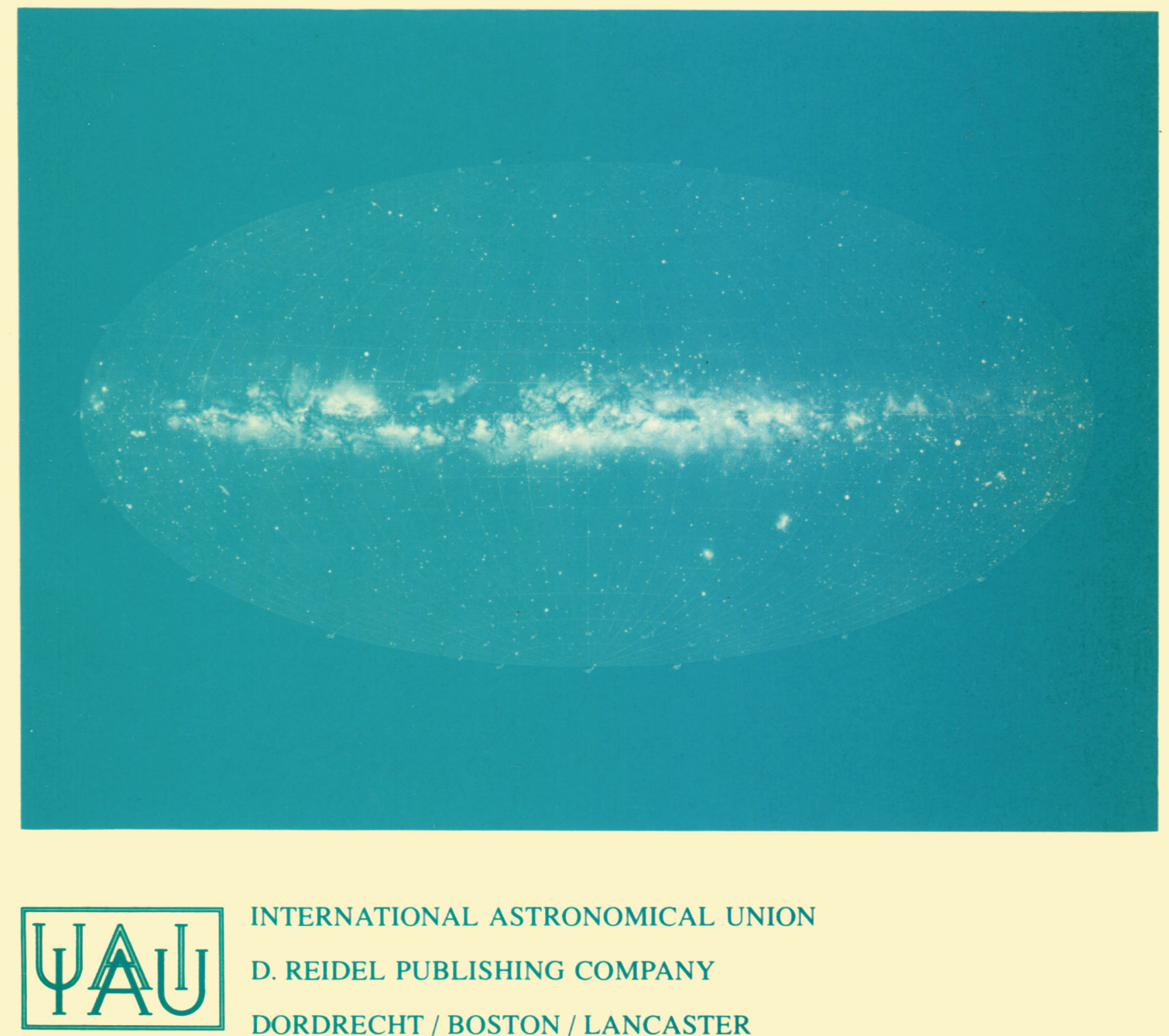

INTERNATIONAL ASTRONOMICAL UNION

D. REIDEL PUBLISHING COMPANY

DORDRECHT / BOSTON / LANCASTER 
THE MILKY WAY GALAXY 


\section{THE MILKY WAY GALAXY}

PROCEEDINGS OF THE 106TH SYMPOSIUM OF THE INTERNATIONAL ASTRONOMICAL UNION HELD IN GRONINGEN, THE NETHERLANDS 30 MAY - 3 JUNE, 1983

EDITED B Y

HUGO VAN WOERDEN

and

RONALD J. ALLEN

Kapteyn Institute, Groningen, The Netherlands

and

W. BUTLER BURTON

Sterrewacht Leiden, The Netherlands

\section{REIDEL PUBLISHING COMPANY \\ A MEMBER OF THE KLUWER ACADEMIC PUBLISHERS GROUP DORDRECHT / BOSTON / LANCASTER}

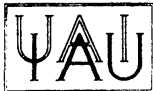


Library of Congress Cataloging in Publication Data

Main entry under title:

The Milky Way Galaxy.

At head of title: International Astronomical Union,

Union Astronomique Internationale.

Includes index.

1. Milky Way-Congresses. I. Woerden, Hugo van.

II. Allen, Ronald J. III. Burton, W. B. (William Butler),

1940- IV. International Astronomical Union.

$\begin{array}{llll}\text { QB857.7.M55 } & 1985 & 523.1^{\prime} 13 & 84-26294\end{array}$

ISBN 90-277-1919-5

ISBN 90-277-1920-9 (pbk.)

Published on behalf of

the International Astronomical Union

by

D. Reidel Publishing Company, P. O. Box 17, 3300 AA Dordrecht, Holland

\author{
All Rights Reserved \\ (C) 1985 by the International Astronomical Union
}

Sold and distributed in the U.S.A. and Canada

by Kluwer Academic Publishers,

190 Old Derby Street, Hingham, MA 02043, U.S.A.

In all other countries, sold and distributed

by Kluwer Academic Publishers Group,

P. O. Box 322, 3300 AH Dordrecht, Holland

No part of the material protected by this copyright notice may be reproduced or utilized in any form or by any means, electronic or mechanical, including photocopying, recording or by any information storage and retrieval system, without written permission from the publisher

Printed in The Netherlands 
TABLE OF CONTENTS

$$
(\mathrm{R}=\text { Review, } \mathrm{I}=\text { Invited Paper })
$$

Preface

Editorial Notes

Committees and Sponsors

List of Participants

xix

Coloured Illustrations

\section{PART I}

HISTORY OF GALACTIC RESEARCH

The Milky Way from antiquity to modern times (R)

$$
\text { M.A. Hoskin }
$$

Kapteyn and statistical astronomy ( $R$ )

$$
\text { E.R. Pau1 }
$$

Studies of the Milky Way 1850-1930: some highlights (R)

$$
\text { R.W. Smith }
$$

The discovery of the spiral arms of the Milky Way (R)

$$
0 \text {. Gingerich }
$$

PART II

COMPOSITION, STRUCTURE AND KINEMATICS

II.1. Galactic Constants, Rotation and Mass Distribution

Models of the mass distribution of the Galaxy (R)

A model of our Galaxy (I)

U. Haud, M. Jôeveer and J. Einasto

Mass models of three southern late-type dwarf spirals

C. Carignan

The Galactic constants - an interim report (I)

F.J. Kerr

Galactic rotation outside the solar circle 
Young galactic clusters and the rotation curve of our Galaxy

J. Hron and H.M. Maitzen

How well do we know the rotation curve of our Galaxy?

P. Pişmiş

Galactic rotation and velocity fields

W.L.H. Shuter and A. Gill

II.2. The Ste1lar Component

The old population ( $R$ )

K.C. Freeman

Near-infrared studies of the Milky Way

H. Okuda

Infrared scanning of the Galactic bulge

R.M. Catchpole, P.A. Whitelock and I.S. Glass

Infrared studies of the stellar population in Baade's Window

R.A. Ruelas-Mayorga, A.R. Hyland and T.J. Jones

On the nature of $\mathrm{OH} / \mathrm{IR}$ stars

D. Engels

The stellar disk component: distribution, motions, age and stellar composition ( $R$ )

G. Lynga

Computer-based catalogue of open-cluster data

G. Lyngå

The kinematics of nearby stars and large-scale radial motion in the Galaxy

$$
\text { S.V.M. Clube }
$$

The Galactic radial gradient of velocity dispersion

M. Mayor and E. Oblak

Stellar chemical-abundance gradient in the direction of the South

Galactic Pole - preliminary results

Ch.F. Trefzger, J.W. Pe1 and A. Blaauw

Star counts, local density and $\mathrm{K}_{\mathrm{z}}$ force (I)

B. Strömgren

The stellar distribution in the Galactic spheroid

G. Gilmore 
Studies of 0-F5 stars at the Galactic poles

R.W. Hilditch, A.D. McFadzean, G. Hill and J.V. Barnes

Galaxy population structure from proper motions

N. Reid and C.A. Murray

II.3. The Gaseous Component: Large-Scale Distribution

Large-scale distribution and motions of gas

Survey of Galactic HI emission at $|\mathrm{b}| \leqq 20^{\circ}$

W.B. Burton and P. te Lintel Hekkert

The vertical distribution of Galactic HI: the Arecibo-Green

Bank Survey

T.M. Bania and F.J. Lockman

New light on the corrugation phenomenon in our Galaxy

J.V. Feitzinger and J. Spicker

HI at the outer edge of the Galaxy and its implications for

Galactic rotation

P.D. Jackson

The electron density in the plane of the Galaxy

J.M. Weisberg, J.M. Rankin and V. Boriakoff

Molecular clouds in external galaxies (I)

J.S. Young

Comments on the distribution of molecules in spiral galaxies

L. J Rickard and P. Palmer

Distribution and motions of $\mathrm{CO}$ in M51

G. Rydbeck, §. Hjalmarson and O. Rydbeck

CO (2-1) observations of Maffei 2

A.I. Sargent, E.C. Sutton, C.R. Masson, T.G. Phillips and

K.-Y. Lo

CO survey of the Southern Milky Way (I)

R.S. Cohen, P. Thaddeus and L. Bronfman

Distribution of $\mathrm{CO}$ in the Southern Milky Way and large-scale structure in the Galaxy

W.H. McCutcheon, B.J. Robinson, R.N. Manchester and

J.B. Whiteoak 
A CO (2-1) survey of the Southern Milky Way

H. van de Stadt, F.P. Israel, Th. de Graauw, C.P. de

Vries, J. Brand, H.J. Habing and J. Wouterloot

The carbon monoxide distribution in the inner Galaxy

T.M. Bania

Outer-Galaxy molecular clouds

M.L. Kutner and K.N. Mead

$\mathrm{CH}$ in the Galaxy

L.E.B. Johansson

High-energy gamma rays and the large-scale distribution of gas and cosmic rays (I)

W. Hermsen and J.B.G.M. Bloemen

Large-scale mapping of the Galaxy by IRAS (I)

T.N. Gautier and M.G. Hauser

II.4. The High-Energy Component

High-energy Galactic phenomena and the interstellar medium (R)

C.J. Cesarsky

The high-latitude distribution of Galactic gamma rays and possible evidence for a gamma-ray halo

A.W. Strong

On the ultraviolet background radiation of the Galaxy

A. Zvereva, A. Severny, L. Granitsky, G. Courtès,

P. Cruvellier and C.T. Hua

Radio continuum emission of the Milky Way and nearby galaxies (I)

R. Beck and W. Reich

Loop I (the North Polar Spur) region - a quasi radio halo

J. Milogradov-Turin

The magnetic-field structure and dynamics of NGC 253

M. Urbanik, U. Klein, R. Beck and R. Wielebinski

Modelling the Galactic contribution to the Faraday rotation of radiation from extra-galactic sources

B.J. Brett

A bisymmetric spiral magnetic field in the Milky Way

Y. Sofue and M. Fujimoto 
Spiral structure of the Milky Way and external galaxies ( $R$ )

$$
\text { D.M. E1megreen }
$$

Which kind of spiral structure can fit the observed gradient of vertex deviation?

E. Oblak and M. Crézé

Spiral structure and kinematics of HI and HII in external galaxies (I)

$$
\text { R.J. Allen, P.D. Atherton and R.P.J. Tilanus }
$$

A systematic study of M81

F. Bash

Determination of Galactic spiral structure at radiofrequencies ( $R$ ) H.S. Liszt

Giant clouds and star-forming regions as spiral-arm tracers B.G. E1megreen

The largest molecular complexes in the first Galactic quadrant

T.M. Dame, B.G. Elmegreen, R.S. Cohen and P. Thaddeus

Interpretation of the apparent anomalies of the Galactic structure T. Jaakkola, N. Holsti and P. Teerikorpi

II.6. Sma11-Scale Structure and Star Formation

Sma11-scale structure and motions in the interstellar gas (I) J.M. Dickey

Atomic hydrogen towards 3C10

J.S. Albinson

Interferometric observations of the sma11-scale structure of Galactic neutral hydrogen

J. Crovisier and J.M. Dickey

A $10-\mathrm{GHz}$ radio-continuum survey of the Galactic-plane region at the Nobeyama Radio Observatory - A complex region at $l=22^{\circ}-25^{\circ}$ Y. Sofue, H. Hirabayashi, K. Akabane, M. Inoue, T. Handa and N. Nakai

Interstellar sodium and Galactic structure. A high-resolution survey

$$
\text { E. Maurice, A. Ardeberg and H. Lindgren }
$$


Molecular-cloud clusters and chains

D.B. Sanders, D.P. Clemens, N.Z. Scoville and P.M. Solomon 329

Three large molecular complexes in Norma

L. Bronfman, R.S. Cohen, P. Thaddeus and H. Alvarez

Comparison of $\mathrm{CO}$ in the Galaxy and the Magellanic Clouds

F.P. Israe1, Th. de Graauw, H. van de Stadt and

C.P. de Vries

Star formation in the Orion Arm (I)

A. Blaauw

A survey of molecular clouds associated with young open star clusters

D. Leisawitz and F. Bash

A new study of the neutral hydrogen in Gould's Belt

T.J. Sodroski, F.J. Kerr and R.P. Sinha

II.7. The Galactic Nucleus

The Galactic Nucleus (R)

J.H. Oort

Fine structure of molecular clouds within 1 minute of arc of the Galactic Center

N. Kaifu, J. Inatani, T. Hasegawa and M. Morimoto

Recombination-1ine observations of the Galactic Centre

J.H. van Gorkom, U.J. Schwarz and J.D. Bregman

Are spiral nuclei "active" in the radio continuum?

P.T.P. Ho and J.L. Turner

Phenomena at the Galactic Centre - a massive black hole? (I) M.J. Rees

II.8. Outskirts and Environment

Highlights of high-velocity clouds (R)

H. van Woerden, U.J. Schwarz and A.N.M. Hulsbosch

A deep survey for high-velocity clouds

A.N.M. Hulsbosch

409

A survey for high-velocity clouds in the inner Galaxy

I.F. Mirabel and R. Morras 
Observations of high-velocity clouds colliding in the anticenter

R. Morras and I.F. Mirabel

The Milky Way: a halo, a corona, or both? (I) K.S. de Boer

II.9. Comparison of Andromeda and Milky Way Galaxies

A comparison of the Andromeda and Milky Way galaxies ( $R$ )

P.W. Hodge

Radial distributions of constituents in M33, the Galaxy, and M51 E.M. Berkhuijsen and U. Klein

A coordinated radio and optical survey of M31

R.A.M. Walterbos and R.C. Kennicutt

Distribution and motions of HI in M31 (I)

E. Brinks and W.B. Burton

The production of a $16-\mathrm{mm}$ film of M31

G.S. Shostak and E. Brinks

Distribution and motion of $\mathrm{CO}$ in M31 (I)

A.A. Stark

Large-scale maps of M31 at middle and far infrared wavelengths (I) H.J. Habing

PART III

DYNAMICS AND EVOLUTION

III.1. Milky Way, Mage1lanic System and Local Group

S1ippery evidence on masses in the Local Group ( $R$ )

D. Lynden-Be11

A collision between the Large and Small Magellanic Clouds $2 \times 10^{8}$ years ago (I)

M. Fujimoto and T. Murai

Tidal interactions between the Galaxy and the Magellanic Clouds N. Ramamani, T. M. Singh and S.M. Alladin

III.2. Dynamics of the Disk

Dynamical evolution of the Galactic disk (R) R. Wielen and B. Fuchs 
Evolution of the vertical structure of galactic disks

J.V. Villumsen

Heating of stellar disks by massive gas clouds

$$
\text { C.G. Lacey }
$$

Stellar velocity distribution in the presence of scattering massive clouds

$$
\text { W. Renz }
$$

Warps and heavy halos

$$
\text { L.S. Sparke }
$$

3-dimensional particle simulation of vertical oscillations in gas discs

$$
\text { T.C. Johns and A.H. Nelson }
$$

Two-fluid gravitational instabilities in a galactic disk

$$
\text { C.J. Jog }
$$

Collective phenomena in a multi-component gravitating system (I) A.M. Fridman, A.G. Morozov, J. Palous, A. Piskunov and V.L. Polyachenko

\section{III.3. Spiral Structure and Star Formation}

Formation and maintenance of spiral structure in galaxies ( $R$ ) C.C. Lin and G. Bertin

Two-dimensional calculations of tightly wound spiral shocks

A.H. Nelson, T.C. Johns and M. Tosa

Oscillations of rotating gas disks: p-modes, g-modes, and $\mathrm{r}$-modes M. Iye

The stabilizing effects of haloes and spiral structure

H. Robe

New $\mathrm{N}$-body experiments on the spiral structure of galaxies

$$
\text { K.0. Thielheim and H. Wolff }
$$

Velocity dispersion and the stability of galactic disks

$$
\text { J. Kormendy }
$$

Periodic orbits relevant for the formation of inner rings in barred galaxies 
On the 3-kpc arm: waves excited at the resonance by an oval distortion in the central region

C. Yuan

A barred galaxy: the inside viewpoint

$$
\text { G.D. van Albada }
$$

Stochastic star formation and spiral structure (I)

P.E. Seiden

Spiral structure in galaxies: large-scale stochastic selforganization of interstellar matter and young stars

$$
\text { J.V. Feitzinger }
$$

Star formation in molecular clouds (I)

F.H. Shu

Star formation in a density-wave-dominated, cloudy interstellar medium

W.W. Roberts Jr. and M.A. Hausman

Spiral tracers and prestellar incubation periods in a cloudy interstellar medium

M.A. Hausman and W.W. Roberts Jr.

III.4. Chemical Composition and Evolution of the Disk

Chemical composition of interstellar material (R)

S.R. Pottasch

Interstellar nịtrogen isotope ratios

R. Güsten and H. Ungerechts

The chemical evolution of the Galaxy (R)

B.A. Twarog

Kinematical and chemical evolution of the Galactic disk C.G. Lacey and S.M. Fa11

III.5. The O1d Population

The formation and early evolution of the Milky Way (R)

S.M. Fal1

Supposed history of our Galaxy

W. Iwanowska

Secular evolution in galaxies

A. May, C.A. Norman and T.S. van Albada 
Gas dynamics and disk-galaxy evolution (I)

R.G. Car1berg

PART IV

LIFE IN THE GALAXY

Life in the Galaxy? (I.)

G.S. Shostak

PART V

SUMMARY AND OUTLOOK

The Milky Way: summary and outlook ( $R$ )

J.P. Ostriker

Concluding general discussion

Subject Index

Object Index

Name Index 